\title{
Highly Ti-loaded MCM-41: Effect of the metal precursor and loading on the titanium distribution and on the catalytic activity in different oxidation processes
}

\author{
José Iglesias*a ${ }^{\mathrm{a}}$ Juan A. Melero ${ }^{\mathrm{b}}$ and Manuel Sánchez-Sánchez ${ }^{\mathrm{c}}$
}

${ }^{a}$ Department of Chemical and Energy Technology, ESCET, Universidad Rey Juan Carlos, C/ Tulipán s/n, 28933 Móstoles, Madrid, Spain

${ }^{b}$ Department of Chemical and Environmental Technology, ESCET, Universidad Rey Juan Carlos, C/ Tulipán s/n, 28933 Móstoles, Madrid, Spain

${ }^{c}$ Instituto de Catálisis y Petroleoquímica, CSIC. C/Marie Curie, 2, Campus de Cantoblanco, E-28049 Madrid. Spain

Published on:

Journal of Molecular Catalysis A: Chemical, 132 (2010) 112-120

doi:10.1016/j.micromeso.2010.02.006

* To whom correspondence should be addressed.

jose.iglesias@urjc.es

Tel. +3491488 7082. Fax +34914887068 


\begin{abstract}
Ti-MCM-41 materials have been prepared by co-condensation of tetraethylorthosilicate with two different titanium sources: titanocene dichloride and titanium isopropoxide. The hydrophobic nature of cyclopentadienyl rings has been used to enhance the interaction between the titanocene dichloride starting compound and the surfactant micelles leading to, after calcination, titanium centers finally located in the surface of the mesopores. In this way, increasing the content of titanocene dichloride in the synthesis gel causes the formation of titanium dioxide nanofibers inside the mesoporous system - clearly visible by TEM analysis for high metal loadings. Such Ti distribution, which has not been observed when using titanium isopropoxide, confers to these Ti-MCM-41 materials a different catalytic behavior to that of conventional Ti-MCM-41 materials for a given Ti content. Thus, samples prepared with titanocene dichloride display higher catalytic activity in the epoxidation of 1-octene and in the oxidative bromination of phenol red than samples prepared with titanium isopropoxide. Besides, in both reactions, the maximum of catalytic activity is reached for lower titanium loading when titanocene dichloride is the starting $\mathrm{Ti}$ source as a consequence of a better accessibility of reactants to the final Ti centres.
\end{abstract}

Keywords: Titanium, MCM-41, $\mathrm{TiO}_{2}$ nanofibers, epoxidation, oxidative bromination 


\section{Introduction}

During the last decades, the synthesis of titanium-containing silica based materials has been a matter of extensive research due to the great potential of these materials in catalytic oxidation processes [1]. The Shell catalyst [2] and the TS-1 zeolite [3], developed during the early seventies and eighties respectively, are two of the most successful examples in industrial applications and the starting points in the development of a numerous family of titanium containing silica-based catalysts, comprising from amorphous silica gels [4] to crystalline zeolites [5]. Further investigations consisted of the incorporation of titanium onto well structured silicas possessing large pores [6,7], such as the series of mesostructured M41S [8] or SBA [9] families. The main aim of these works was to overcome the diffusion problems of the zeolitc micropores, which severely limits their catalytic application. In this sense, the development of the new mesostructured materials during nineties opened new opportunities to generate silica-based catalysts containing titanium as active redox sites. The first step in this research was carried out by Corma et al. [6], who reported the synthesis of a titaniumcontaining MCM-41 material through a direct-synthesis procedure. Later on, numerous efforts have been focused on the synthesis and preparation of new titanium-containing silica-based mesostructured materials [10-16]. For this purpose, a large variety of structure directing agents, titanium precursors and synthesis conditions have been investigated, mainly following two different strategies: co-condensation of silicon and titanium precursors $[6,10]$ or postsynthetic functionalization by grafting procedures [17,18]. The first option leads to homogeneously dispersed titanium centers, but they can be buried inside the silica walls so that an important fraction of the loaded metal sites are not accessible. On the contrary, the second strategy ensures the accessibility to the titanium sites but lower metal dispersion degree is achieved. Obviously, the different titanium locations and environments determine their catalytic activity [19]. Combining the advantages of both synthesis procedures, high 
dispersion and accessibility of metal sites, is catalytically desirable. The hydrophilichydrophobic control of the interaction between the titanium source and the surfactant templating mesopores has resulted successful. Thus, the choice of non-ionic surfactants such as alkylene oxides and titanocene dichloride as metal source provokes the interaction between the hydrophobic cyclopentadienyl rings of titanocene with the also-hydrophobic core of the micelle [15,20,21]. This procedure has effectively been extended to other metal species [22], but always for non-ionic surfactants and under acid conditions.

In this work, we have applied a similar approach by using two titanium sources of different hydrophobic character - titanocene chloride and titanium tetraisopropoxide - under basic synthesis conditions and using an ionic surfactant (the conventional CTABr). The resultant materials, obtained for a huge $\mathrm{Si} / \mathrm{Ti}$ molar ratio range (from 1 to 160 ), have been carefully characterized by means of different analysis techniques in order to determine the final location of titanium. The possible effect of the different dispersion and accessibility of reactants to titanium sites on their catalytic behaviour has been tested in the epoxidation of 1octene and in the oxidative bromination of phenol red.

\section{Experimental}

\subsection{Synthesis of Ti-MCM-41 materials}

Titanium-contained mesoporous MCM-41 molecular sieves were prepared following the method proposed by Pang et al. [23] for the synthesis of pure silica MCM-41 materials, which here is modified by adding titanocene dichloride $\left(\mathrm{Cp}_{2} \mathrm{TiCl}_{2}\right.$, Aldrich) or titanium isopropoxide $\left(\mathrm{Ti}(\mathrm{OiPr})_{4}\right.$, Alfa-Aesar) as metal precursors. In a typical synthesis, $2.5 \mathrm{~g}$ of hexadecyl trimethylammonium bromide (CTABr, Aldrich) and $8 \mathrm{~g}$ of dimethyl amine (DMA, $40 \%$ wt in water, Aldrich) were dissolved in $95 \mathrm{~g}$ of deionised water - milliQ grade - at room 
temperature. The mixture was then homogenized until complete dissolution, and proper amounts of the titanium and silicon sources (TEOS, Aldrich) were then added. The amount of the titanium precursor was increased to give gels with Si/Ti molar ratios between 160 and 1 . The molar composition of the synthesis gel was: $120 \mathrm{H}_{2} \mathrm{O}: 1.5 \mathrm{DMA}: 0.15 \mathrm{CTABr}: 1 \mathrm{SiO}_{2}$ : $x \mathrm{TiO}_{2}$, where $x$ was varied from 0.00625 to 1 . After the addition of the silicon precursor, the suspension was stirred for 4 additional hours before being hydrothermally aged at $110{ }^{\circ} \mathrm{C}$ for 48 hours in a PTFE-lined stainless-steel autoclave under static conditions. The material was then recovered by filtration and dried overnight at room temperature. The removal of the surfactant occluded in mesopores was carried out by calcination in static air conditions at 550 ${ }^{\circ} \mathrm{C}$ during 5 hours.

The samples have been called Ti-MCM-41-Cp-X or Ti-MCM-41-OR-X for the materials prepared with titanocene dichloride and titanium isopropoxide respectively, where $\mathrm{X}$ denotes the Si/Ti molar ratio of the starting gel.

\subsection{Characterization of the samples}

Textural properties were evaluated by nitrogen adsorption-desorption isotherms performed at $-196^{\circ} \mathrm{C}$ in a TriStar Unit (Micromeritics). Specific surface area values were estimated by B.E.T. method and the total pore volume was assumed to be that recorded at $\mathrm{p} / \mathrm{p}_{0}=0.985$ for each sample. Pore sizes distributions were calculated through the B.J.H. method applying the K.J.S. correction and using the adsorption branch values of the isotherms. X-ray diffraction patterns were collected on a Philips X'pert unit fitted with an accessory for low angle experiments, using the $\mathrm{K}_{\alpha}$ line of copper and in two different $2 \theta$ ranges from $0.5^{\circ}$ to $10^{\circ}$ for the evaluation of the mesoscopic ordering, and from 5 to $50^{\circ}$ when searching the presence of crystalline titanium dioxide phases. In both cases, the assays were recorded with a step size of $0.01^{\circ}$. Diffuse Reflectance UV-VIS spectra (DR UV-VIS) were recorded under ambient conditions on a VARIAN CARY-500 spectrophotometer equipped with a diffuse reflectance 
accessory in the wavelength range from $200 \mathrm{~nm}$ to $600 \mathrm{~nm}$. Transmission electron microscopy (TEM) microphotographs were collected on a PHILIPS TECNAI-20 electron microscope operating at $200 \mathrm{kV}$. Raman spectra were recorded at room temperature using a LabRam HR spectrometer fitted with a Jobin-Yvon CCD detector and an excitation line at $632.8 \mathrm{~nm}$ from an Helium Neon laser (source power: $11 \mathrm{~mW}$ ). Titanium content was determined by ICPatomic emission spectroscopy. The samples $(100 \mathrm{mg})$ were dissolved in aqueous hydrofluoric acid. After dissolution, the sample was transferred into $1 \mathrm{~L}$ calibrated flask and diluted with water. An absorption standard solution of $\mathrm{Ti}(1000 \mu \mathrm{g}$ ml-1 in water) was used for the calibration of the equipment.

\subsection{Catalytic tests}

The catalytic epoxidation of 1-octene was carried out in a batch multi reactor equipped with a temperature controller at $80^{\circ} \mathrm{C}$ under stirring for 6 hours. Catalysts and 1-octene (catalyst : 1octene mass ratio of 0.02 ; mass catalyst of $0.5 \mathrm{~g}$ ) were charged into the reactor flasks and the system was warmed up to the reaction temperature with a heating rate of $10^{\circ} \mathrm{C} \cdot \mathrm{min}^{-1}$. Once the reaction temperature $\left(80^{\circ} \mathrm{C}\right)$ was reached, the required amount of oxidant was added. Tertbutyl hydroperoxide (TBHP; 5,5 $\mathrm{M}$ in hexane, Fluka) was used as oxidant (TBHP : 1-octene molar ratio of 0.8 ). Then, aliquots were withdrawn each hour during the reaction course with the purpose of monitoring the evolution of the oxidant conversion and oxidant selectivity towards the epoxide formation - efficiency in the use of the oxidant -. Peroxide conversion was measured by iodometric titration whereas reaction products were analyzed by means of gas chromatography analysis.

The oxidative bromination of phenol red was carried out using the method described by Stucky et al [24]. In a typical reaction, a $0.1 \mathrm{~g}$ of titanium-contained MCM-41 material was suspended in $50 \mathrm{ml}$ of an aqueous solution of phenol red $5 \mathrm{mM}$, containing $\mathrm{KBr}(0.1 \mathrm{M})$ and $\mathrm{H}_{2} \mathrm{O}_{2}(0.1 \mathrm{M})$. The resultant suspension was stirred for 6 hours at room temperature and an 
aliquot was withdrawn from the reaction solution each hour. The sample was then filtered in a syringe filter and analyzed by colorimetric analysis in a Jasco V-630 spectrophotometer. Mass balance was assessed by recording the absorbance at $432 \mathrm{~nm}$ for the calculus of phenol red conversion and at $591 \mathrm{~nm}$ for the quantification of the yield towards bromo-phenol blue, which was the unique detected product. Aliquots were withdrawn from the reaction mixture for monitoring the evolution of the catalytic tests.

\section{Results and discussion}

Ti-MCM-41 materials have been prepared using two different titanium sources, titanocene dichloride $-\mathrm{Cp}_{2} \mathrm{TiCl}_{2}-$ and titanium isopropoxide $-\mathrm{Ti}(\mathrm{OiPr})_{4}-$ for comparison studies. For the synthesis of the mesostructured materials, the method proposed by Pang et al. [23] has been employed since the use of dialkyl amines instead of alkaline inorganic salts as mineralization agents allows a better control of $\mathrm{pH}$. Besides, avoiding the use of strong alkaline salts, such as $\mathrm{NaOH}$, removes the interferences of the alkaline cations in the incorporation of the titanium species within the silica matrix.

Table 1 lists the physico-chemical properties of titanium-functionalized MCM-41 materials prepared with titanocene dichloride or titanium isopropoxide as metal precursors. Both titanium sources gave almost quantitative incorporation of the metal species onto the calcined materials, which have titanium loadings from 0.5 to about $35 \%$ wt. when the samples are obtained from starting gels with $\mathrm{Si} / \mathrm{Ti}$ ratio of 160 to 1 , respectively. The Ti-MCM-41 samples are almost systematically richer in $\mathrm{Ti}$ than their corresponding synthesis gels, suggesting that the silicon species could not completely be incorporated in the final solids under the basic synthesis conditions. 
Textural and structural properties estimated from nitrogen adsorption-desorption isotherms and X-ray diffraction patterns, respectively, reveal some differences between materials with different titanium sources, more evident at higher Ti content. Thus, for low titanium loadings, the surface area, total pore volume and mean pore size are rather similar regardless the metal source. Increasing the titanium content leads to a systematic decrease in all the textural properties, more evident in surface area and pore volume rather than in mean pore size, irrespective of the used $\mathrm{Ti}$ source. Indeed, the mean pore size is scarcely diminished. Differences become larger when higher loadings of the metal precursor are employed and also when titanium isopropoxide is the Ti source, so that the Ti-MCM-41-OR-1 sample is almost not porous. Its homologue sample prepared with titanocene, Ti-MCM-41-Cp-1, possesses a notable mesoporosity (surface area of $350 \mathrm{~m}^{2} \mathrm{~g}^{-1}$ and pore volume of $0.57 \mathrm{~cm}^{3} \mathrm{~g}^{-1}$ ).

These differences are more explicitly shown in Figure 1, which depicts the nitrogen adsorption desorption isotherms for all materials. The materials prepared from gels of Si/Ti ratios higher than 5 show type IV isotherms, accordingly to the IUPAC classification. This involves narrow pore sizes distributions - Figure 2 - and well ordered mesostructures. Moreover, the steep adsorption slope caused by the capillary condensation of nitrogen inside the mesopores, is located at the same relative pressure (at $c a . \mathrm{p} / \mathrm{p}_{0}=0.33$ ) in every sample, irrespective of the titanium loading. As above mentioned, the reduction in the adsorbed volume for Ti-richest materials prepared with titanium alkoxide is more drastic than for those prepared with titanocene dichloride. Indeed, the isotherm of the material prepared with the maximum loading in titanium isopropoxide, Ti-MCM-41-OR-1, is practically flat, whereas the analogue sample synthesized with $\mathrm{Cp}_{2} \mathrm{TiCl}_{2}$ still maintains a significant nitrogen uptake, though their capillary condensation is missed. These results indicate that titanocene dichloride should be used as titanium precursor to obtain better ordered Ti-rich MCM-41 materials, presumably as a consequence of the different chemical behaviour of both Ti sources in the 
hydrolysis and condensation processes under the basic synthesis conditions. $\mathrm{Ti}(\mathrm{OiPr})_{4}$, like other titanium alkoxides, easily hydrolyzes and condenses under basic synthesis conditions, whereas titanocene dichloride, which is a more chemically stable source, is not easily hydrolyzed. Therefore, the condensation rate of titanocene species is delayed, matching that of silica and producing better ordered materials.

All the above mentioned indications are supported by X-ray diffraction studies. Figure 3 displays the XRD patterns of Ti-contained MCM-41 materials using (A) $\mathrm{Cp}_{2} \mathrm{TiCl}_{2}$ or (B) $\mathrm{Ti}(\mathrm{OiPr})_{4}$ as $\mathrm{Ti}$ source. At low titanium loadings $-\mathrm{Si} / \mathrm{Ti}$ molar ratios higher than $10-$ the samples show diffraction patterns corresponding to a P6mmc planar hexagonal geometry, typical of MCM-41 materials. The diffraction peaks 100, 110, 200 are clearly visible and even the reflection 210 is apparent in XRD patterns of the samples Ti-MCM-41-Cp-X with X higher than 10. The presence of the well-defined long-range diffraction peaks, together with their high intensity, evidence an excellent ordering degree of these materials, which systematically decreases as titanium increases into the synthesis gel, regardless the Ti source. This destructuration became dramatic for materials from gels with Si/Ti molar ratios lower than 5, particularly for Ti-MCM-41-OR ones, in fairly agreement with the $\mathrm{N}_{2}$ adsorption isotherms results and in line with the lower hydrolysis rate expected for titanocene dichloride. However, no great discrepancies have been observed in terms of interplanar spacing calculated by the Bragg's law - or even in pore wall thickness - estimated by subtraction of the unit cell dimension $\left(a_{0}\right)$ and the pore size diameter $\left(D_{p}\right)-($ See Table 1$)$. Thus, it can be concluded that both titanium loading and titanium source nature do not largely influence on the structural parameters of MCM-41 materials, apart from the mesoscopic ordering degree, under the experimental conditions used in this investigation.

In order to assess the presence of bulk crystalline titanium dioxide domains in the final samples, high angle X-ray diffraction patterns were collected - Figure 4 -. Anatase-structured 
$\mathrm{TiO}_{2}$ domains are only present, at the XRD detection level, in samples containing high titanium loadings - samples prepared from gels with Si/Ti of 2 or lower - becoming more evident in Ti-MCM-41-OR samples, where the anatase domains are of larger size as indicated by the relative sharp width of reflections. These results do not prove the absence of $\mathrm{TiO}_{2}$ domains in Ti-poorer samples, since the Ti dispersion could be so high that their particles are not XRD detectable. For this reason, the characterization of the samples has been complemented by other characterization studies.

Figure 5 depicts the DR UV-Vis spectra recorded for the different Ti-MCM-41 materials. All UV spectra are dominated by a strong band at $c a .210 \mathrm{~nm}$, which is more intense in these of the Ti-MCM-41-Cp samples. This band, attributed to the charge transfer from oxygen atoms to $\mathrm{Ti}^{4+}$ has been taken as evidence of $\mathrm{Ti}^{4+}$ tetrahedrally coordinated and of the absence of $\mathrm{Ti}$ O-Ti bonds [25], suggesting a very good dispersion of metal sites in the silica matrixes. Increasing the titanium content induces the appearance of other lower-energy signals at $c a$. 250 and $300-330 \mathrm{~nm}$, assigned to the presence of titanium sites in higher coordination numbers and having Ti-O-Ti bonds; in other words, a higher Ti loading gives rise to a lower titanium dispersion degree. Thus, UV absorption band at $250 \mathrm{~nm}$ is usually ascribed to the presence of isolated penta-coordinated titanium sites or $\mathrm{TiO}_{2}$ oligomers [26], whereas signals located at wavelengths over $300 \mathrm{~nm}$ are attributed to the presence of bulk $\mathrm{TiO}_{2}$ phases. Taking these assignments in mind, the use of titanocene dichloride or titanium isopropoxide sources produces significant differences regarding to the final configuration of titanium in MCM-41 samples. In the case of Ti-MCM-41-Cp samples (Figure 5A), low Ti loadings exclusively produce isolated titanium sites whereas higher coordination $\mathrm{Ti}$ species emerge as titanium content increases. Thus, in the spectra of the samples with $\mathrm{Si} / \mathrm{Ti}$ ratios lower than 40 , a small shoulder at $c a .250 \mathrm{~nm}$ is detected and its intensity increases with the Ti content, becoming the dominant band in the spectra of the samples Ti-MCM-41-Cp-10. For that sample, the Ti 
content would have overcome the critical amount to interact with surfactant micelles induced by the hydrophobic nature of their cyclopentadienyl rings. In this sense, the appearance and the increase of the signal intensity detected at $250 \mathrm{~nm}$ can be associated to the formation of titanium dioxide oligomers having titanium coordination somehow intermediate between the tetrahedral environment of the isolated species and the octahedral coordination typical of bulk titanium dioxide. Further increase of Ti content causes the appearance of a new UV band at wavelengths higher than $300 \mathrm{~nm}$, assignable to bulk titanium dioxide domains. DR-UVvisible spectra of samples prepared with titanium alkoxide (Figure 5B) show high intense bands at $c a .330 \mathrm{~nm}$ even for samples with low Ti contents (obtained from gels with $\mathrm{Si} / \mathrm{Ti}$ ratio of 80 or lower), which can be considered as a probe of the low titanium dispersion degree using this metal source.

TEM images were recorded trying to shed light on the $\mathrm{Ti}$ distribution along the particles. Figure 6 depicts some microphotographs recorded for Ti-MCM-41 materials with different Ti sources and content. Low metal loadings lead to excellent-ordered mesoscopic structures when using titanocene dichloride as precursor, whereas titanium alkoxide leads to the formation of wormlike-type porous materials. These differences become more evident as the titanium loading increases, although the samples prepared with the maximum $\mathrm{Ti}$ content basically lacks any porous order and possesses some $\mathrm{TiO}_{2}$ domains. However, the most evident difference showed by TEM between samples prepared with different Ti sources is the different $\mathrm{Ti}$ species distribution. These differences are particularly illustrative between samples prepared from gels with a $\mathrm{Si} / \mathrm{Ti}$ molar ratio of 5 . Whereas the wormlike structure is still present for the sample Ti-MCM-41-OR-5 and no evidence of bulk titanium dioxide domains is detected, its homologue sample Ti-MCM-41-Cp-5 has some $\mathrm{TiO}_{2}$ nanofibers, which flows along the porous structure, drawing the pore paths. The origin of these titanium oxide fibers could be attributed to the interaction taking place between the hydrophobic 
moiety of CTABr micelles with the cyclopentadienyl rings of titanocene during the material formation process. Such chemical affinity leads to a great $\mathrm{Ti}$ concentration in a particular spatial location. The subsequent surfactant removing by calcination of the material leaves $\mathrm{Ti}$ species onto the inner surface of the mesoscopic porous system. If the titanium loading is high enough, $\mathrm{TiO}_{2}$ chains are formed along the pores. These species could be the $\mathrm{TiO}_{2}$ oligomers responsible for the band detected by DR UV-Vis spectroscopy at $c a .250 \mathrm{~nm}$. Whether this titanium dioxide chain forms just rod-like structures inside the mesopores or tubes covering the surface of them, is not distinguishable through the TEM micrographs collected in this work. However, in the sample Ti-MCM-41-Cp-5, these rod-like $\mathrm{TiO}_{2}$ fibers are presumably hollow and maybe they are decorating the mesopore walls, as their massive presence (Figure 6) does not provoke a so substantial decrease of the textural properties of the sample (Table 1). In any case, what is unquestionable is that the use of titanocene dichloride leads to very accessible titanium species supported onto MCM-41 materials and, if the metal loading is enough, high surface area titanium dioxide particles supported on silica can be achieved when prepared through a co-condensation pathway.

In order to check the presence and nature of titanium dioxide domains in titanocene dichloride-based MCM-41 materials, Raman spectra were recorded (Figure 7). For comparison purposes, the spectrum corresponding to anatase has also been included in Figure 7, giving bands at 641,517 and $398 \mathrm{~cm}^{-1}$. Raman spectrum of any silica-based material displays signals located at 288 and $450 \mathrm{~cm}^{-1}$, which could be attributed to different siloxane bondings [27]. Signals attributable to anatase are not detected in the spectra of our Ti-MCM41-Cp sample unless the metal loading reaches a Si/Ti molar ratio of 2 in the starting gel, in good agreement with the high-angle XRD patterns (Figure 4) and with TEM pictures (figure 6). The spectra of every sample have an extra band at $697 \mathrm{~cm}^{-1}$, which has been attributed to the presence of Ti-O-Ti bonds [28]. Therefore, even for low Ti loaded samples, such as Ti- 
MCM-41-Cp-20,,Ti species containing Ti-O-Ti exist. This indication by Raman spectroscopy, together with the TEM, suggests that the Ti dispersion is not as high as it could be inferred from DR UV-Vis spectroscopy for the materials prepared using $\mathrm{Cp}_{2} \mathrm{TiCl}_{2}$. The reason for the formation of Ti-O-Ti linkages is that titanium precursor reaches high concentration around the surfactant micelle during the formation of the material. It supports the beneficial effect of using titanocene dichloride as $\mathrm{Ti}$ source to lead highly metal accessibility onto MCM-41 materials prepared through a co-condensation pathway.

The influence of the nature of Ti precursors and $\mathrm{Ti}$ content of Ti-MCM-41 materials was tested in the epoxidation of 1-octene with tert-butyl hydroperoxide (TBHP) and the oxidative bromination of phenol red to bromophenol blue.

Figure 8 compares the 1-octene conversion and the TBHP efficiency - selectivity of the oxidant towards the oxirane - for Ti-MCM-41 materials prepared from gels with different $\mathrm{Si} / \mathrm{Ti}$ ratios and with different $\mathrm{Ti}$ sources. All materials show catalytic activity, being the substrate conversion in the range $20-40 \%$ for the samples Ti-MCM-41-Cp and slightly lower for Ti-MCM-41-OR ones. The efficiency of the oxidant also reaches higher values for catalysts Ti-MCM-41-Cp probably because of their lower amount of bulk titanium dioxide domains - usually responsible for the non-oxidative decomposition of hydroperoxide [29] -. On the other hand, the catalytic activity of Ti-MCM-41 materials is also influenced by their titanium content. For both series of catalysts, a maximum in the catalytic activity for intermediate titanium contents was found, as a consequence of the counteracted influence on the conversion of the number of the isolated active sites and the formation of species possessing Ti-O-Ti bonds. The maximum of the catalytic activity for the series of materials Ti-MCM-41-Cp and Ti-MCM-41-OR were found for $\mathrm{Si} / \mathrm{Ti}$ ratios of 40 and 20, respectively. Therefore, the optimal titanium loading is lower for materials prepared with titanocene dichloride than for these prepared with titanium alkoxide. This fact, together with their overall 
higher catalytic activity - higher substrate conversion as well as efficiency in the use of the oxidant - suggests and supports the above mentioned better accessibility to titanium centres in Ti-MCM-41-Cp materials. The hydrophobic nature of cyclopentadienyl rings enhances the interaction of titanium species with the surfactant micelles, making the resultant titanium sites readily accessible when the surfactant is removed. However, high titanium concentration in the mesopores leads to a quicker saturation of titanium species and to the consequent formation Ti-O-Ti linkages, which reduces their catalytic activity not only per active centre but also in absolute terms, partially helped by the corresponding decrease in surface area.

The oxidative bromination of phenol red with $\mathrm{KBr}$ in presence of $\mathrm{H}_{2} \mathrm{O}_{2}$ was also used as catalytic test. The most significant results are depicted in Figure 9. The bromination reaction is initiated by the activation of hydrogen peroxide by the active centre to oxidize the bromide ion to hypobromous acid and subsequently followed by the reaction with phenol red to lead bromophenol blue [24]. The maximum of the catalytic activity for this reaction was found for Ti-richer samples along the both series of samples (Figure 9, samples Ti-MCM-41-Cp-10 and Ti-MCM-41-OR-2, respectively) than for the epoxidation of 1-octene (Figure 8, samples TiMCM-41-Cp-40 and Ti-MCM-41-OR-20). It is particularly relevant the difference in Ti content of the samples given the maximum conversion. Therefore, the sense of this shift along $\mathrm{Si} / \mathrm{Ti}$ ratio values is independent on the nature of titanium precursor, and suggests that the presence of partially condensed titanium dioxide oligomers does not reduce the activity of TiMCM-41 materials in peroxidative bromination.

For comparison purposes, the catalytic activity of the different Ti-MCM-41 samples on both the catalytic epoxidation of 1-octene with TBHP and the oxidative bromination of phenol red versus Ti atom content per surface area, has been displayed in Figure 10. For a giving Ti loading per surface area, higher conversion of substrates are achieved for samples Ti-MCM41-Cp, which is in agreement with the higher accessibility of titanium species in these 
samples. On the other hand, this Figure makes clearer the different optimal titanium content in both series of MCM-41 materials in both catalytic tests. Thus, Ti-richer samples are ideally required for the oxidative bromination of phenol red than for epoxidizing 1-octene. This result contrasts with those of previous works in bromination [21,29], which claimed that isolated titanium sites are the most catalytic species. However, these discrepancies could be justified considering that titanium-containing mesostructured materials reported by Thanabodeekij et al. [30] contain an important amount of $\mathrm{TiO}_{2}$ oligomers according with their DR UV-Vis spectra. Therefore, the catalytic activity of isolated titanium species in the activation of hydrogen peroxide could be supported by the presence of Ti oligomers. In fact, since the maximum catalytic activity observed within this work corresponds to samples having some Ti-O-Ti linkages (Figure 7), and a negligible catalytic activity is found for low metal loadings, where the titanium species seems to be incorporated as isolated tetrahedral sites, it seems that there is a cooperative effect between close titanium sites that enhances catalytic activity in oxidative bromination. An excessive amount of titanium causes the reduction of the overall catalytic activity, probably because of both the very low catalytic activity of the bulk titanium dioxide and the surface area decrease of the catalysts with the increase of Ti content (Table 1). 


\section{Conclusions}

The synthesis of well structured Ti-MCM-41 materials can be easily accomplished both from titanocene dichloride and titanium isopropoxide as metal precursors. However, the hydrophobic nature of cyclopentadienyl rings around the metal centre in titanocene and its low hydrolysis-condensation rate allows controlling the interaction with surfactant micelles to lead highly accessible titanium sites onto the final materials. This behaviour has been probed by the formation of titanium dioxide nanofibers inside the mesopores of the Ti-MCM-41 materials prepared with titanocene as Ti source, particularly evident for Ti-MCM-41 prepared from gels with a $\mathrm{Si} / \mathrm{Ti}$ ratio of 5 . This difference in chemical behaviour of titanocene dichloride and titanium isopropoxide determines the catalytic activity of Ti-MCM-41 materials in oxidation reactions. Although both kinds of materials show remarkable catalytic activity in olefin epoxidation and peroxidative bromination of phenol red, it is clearly higher in materials prepared with titanocene and, besides, the optimum catalytic behaviour is reached with lower Ti content than for materials prepared with the titanium alkoxide. 


\section{$\underline{\text { References }}$}

[1] The terms "Titanium + catalytic oxidation" provides 2137 entries in a search performed in ISI Web of Knowledge.

[2] F. Wattimena, H.P. Wulff, Shell Oil, Br. Pat. 1,249,079 (1971).

[3] M. Taramasso, G. Perego, B. Notari, Snamprogetti S.P.A., US Pat. 4,410,501 (1982).

[4] D.C.M. Dutoit, M. Schneider, A. Baiker, J. Catal. 153 (1995) 165.

[5] M.A. Camblor, A. Corma, A. Martínez, J. Pérez-Pariente, J. Chem. Soc. Chem. Commun. 8 (1992) 589.

[6] A. Corma, M.T. Navarro, J. Pérez-Pariente, J. Chem. Soc. Chem. Commun. 2 (1994) 147.

[7] Z.H. Luan, E.M. Maes, P.A.W. van der Heide, D.Y. Zhao, R.S. Czernuszewicz, L. Kevan, Chem. Mater. 11 (1999) 3680.

[8] J.S. Beck, J.C. Vartuli, W.J. Roth, M.E. Leonowicz, C.T. Kresge, K.D. Schmitt, C.T.W. Chu, D.H. Olson, E.W. Sheppard, S.B. McCullen, J.B. Higgins, J.L. Schlenker, J. Am. Chem. Soc. 114 (1992) 10834.

[9] D.Y. Zhao, Q.S. Huo, J.L. Feng, B.F. Chmelka, G.D. Stucky, J. Am. Chem. Soc. 120 (1998) 6024.

[10] T. Blasco, A. Corma, M.T. Navarro, J. Pérez-Pariente, J. Catal. 156 (1995) 65.

[11] S. Gontier, A. Tuel, Zeolites 15 (1995) 601.

[12] K.A. Koyano, T. Tatsumi, Chem. Commun. 2 (1996) 145.

[13] S.A. Bagshaw, F. DiRenzo, F. Fajula, Chem. Commun. 18 (1996) 2209. 
[14] O.A. Kholdeeva, M.S. Mel'gunov, A.N. Shmakov, N.N. Trukhan, V.V. Kriventsov, V.I. Zaikovskii, M.E. Malyshev, V.N. Romannikov, Catal. Today 91 (2004) 205.

[15] J.A. Melero, J.M. Arsuaga, P. de Frutos, J. Iglesias, J. Sainz, S. Blázquez, Microporous Mesoporous Mater. 86 (2005) 364.

[16] A. Vinu, P. Srinivasu, V.V. Balasubramaniam, K. Ariga, T. Mori, Y. Nemoto, Chem. Lett. 37 (2008) 1016.

[17] T. Maschmeyer, F. Rey, G. Sankar, J.M. Thomas, Nature 378 (1995) 159.

[18] G. Calleja, R. van Grieken, R. García, J.A. Melero, J. Iglesias, J. Mol. Catal. A: Chem. $182-183$ (2002) 215.

[19] L.Y. Chen, G.K. Guah, S. Jaenicke, Catal. Lett. 50 (2004) 107.

[20] M.E. Raimondi, L. Marchese, E. Gianotti, T. Maschmeyer, J.M. Seddon, S. Coluccia, Chem. Commun. 1 (1999) 87.

[21] M.E. Raimondi, E. Gianotti, L. Marchese, G. Martra, T. Maschmeyer, J.M. Seddon, S. Coluccia, J. Phys. Chem. B. 104 (2000) 7102.

[22] M.E. Raimondi, E. Gianotti, L. Marchese, G. Martra, T. Maschmeyer, J.M. Seddon, S. Coluccia, Catal. Lett. 76 (2001) 21.

[23] W. Lin, Q. Cai, W. Pang, Y. Yue, B. Zou, Microporous Mesoporous Mater. 33 (1999) 187.

[24] J.V. Walker, M. Morey, H. Carlsson, A. Davidson, G.D. Stucky, A. Butler, J. Am. Chem. Soc. 119 (1997) 6921.

[25] D.R.C. Huybrechts, P.L. Buskens, P.A. Jacobs, 71 (1992) 129.

[26] J.M. Fraile, J.I. García, J.A. Mayoral, E. Vispe, J. Catal. 233 (2005) 90.

[27] H. Gao, W. Lu, Q. Chen, Microporous Mesoporous Mater. 34 (2000) 307. 
[28] M.S. Morey, S. O’Brien, S. Schwarz, G.D. Stucky, Chem. Mater. 12 (2000) 898.

[29] C.M. Miller, R.L. Valentine, Water Res. 33 (1999) 2805.

[30] N. Thanabodeekij, W. Tanglumlert, E. Gulari, S. Wonkasemjit, Appl. Organomet. Chem. 19 (2005) 1047. 


\section{Figure Captions}

Figure 1. $\mathrm{N}_{2}$ adsorption-desorption isotherms a $77 \mathrm{~K}$ recorded for Ti-MCM-41 materials prepared in presence of (A) titanocene dichloride and (B) titanium isopropoxide. For clarity, increasing quantities have been added to each isotherm (step of $50 \mathrm{cc} \cdot \mathrm{g}^{-1}$ ).

Figure 2. Pore sizes distributions calculated through the BJH-KJS method for Ti-MCM-41 materials prepared from gels with the indicated $\mathrm{Si} / \mathrm{Ti}$ molar ratios and with (A) $\mathrm{Cp}_{2} \mathrm{TiCl}_{2}$ and (B) $\mathrm{Ti}(\mathrm{OiPr})_{4}$ as $\mathrm{Ti}$ sources.

Figure 3. Low angle XRD patterns of Ti-MCM-41-Cp (A) and Ti-MCM-41-OR (B) materials.

Figure 4. High angle XRD patterns collected of Ti-MCM-41-Cp (A) and Ti-MCM-41-OR (B) materials.

Figure 5. DR UV-Vis spectra of Ti-MCM-41-Cp (A) and Ti-MCM-41-OR (B) materials.

Figure 6. TEM microphotographs collected for Ti-MCM-41 materials with different Ti content and with different Ti sources.

Figure 7. Raman spectra recorded of Ti-MCM-41-Cp materials.

Figure 8. 1-octene conversion and TBHP efficiency after 6 hours versus Si/Ti ratio of the series of Ti-MCM-41-Cp (A) and Ti-MCM-41-OR (B).

Figure 9. Catalytic results after 1 hour in the peroxidative bromination of phenol red to bromophenol blue in presence of different Ti-MCM-41 catalysts. (A) Substrate conversion versus $\mathrm{Si} / \mathrm{Ti}$ ratio (B) Evolution of the UV spectrum with time from 0 to 60 minutes for the sample Ti-MCM-41 prepared with a Si/Ti ratio of 10 and titanocene dichloride as Ti source. 
Figure 10. Catalytic activity of Ti-MCM-41-Cp and Ti-MCM-41-OR materials in A) the epoxidation of 1-octene and B) the peroxidative bromination of phenol red to bromophenol blue, versus titanium content per surface area. 

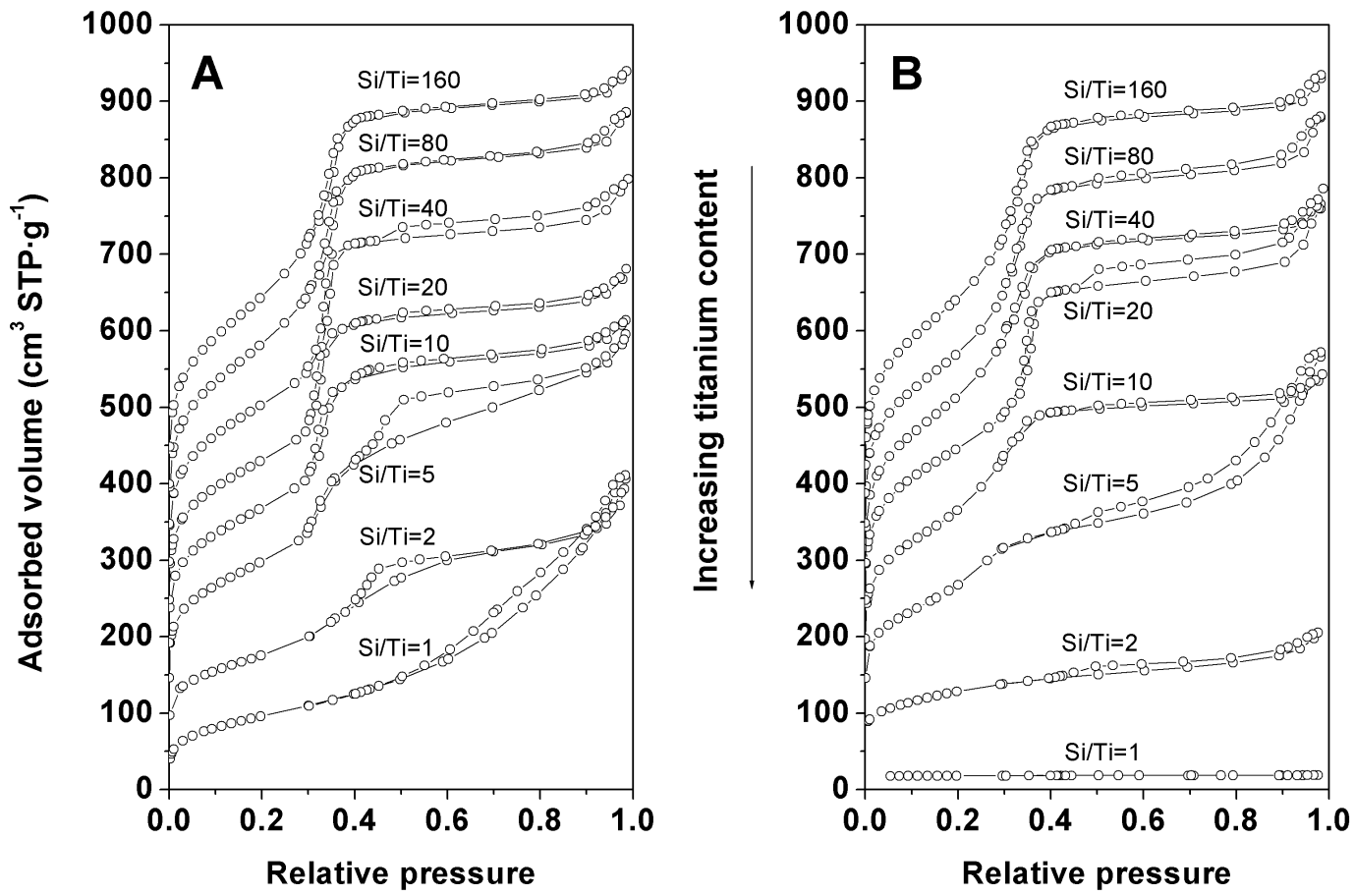

Figure 1

J. Iglesias et al. 


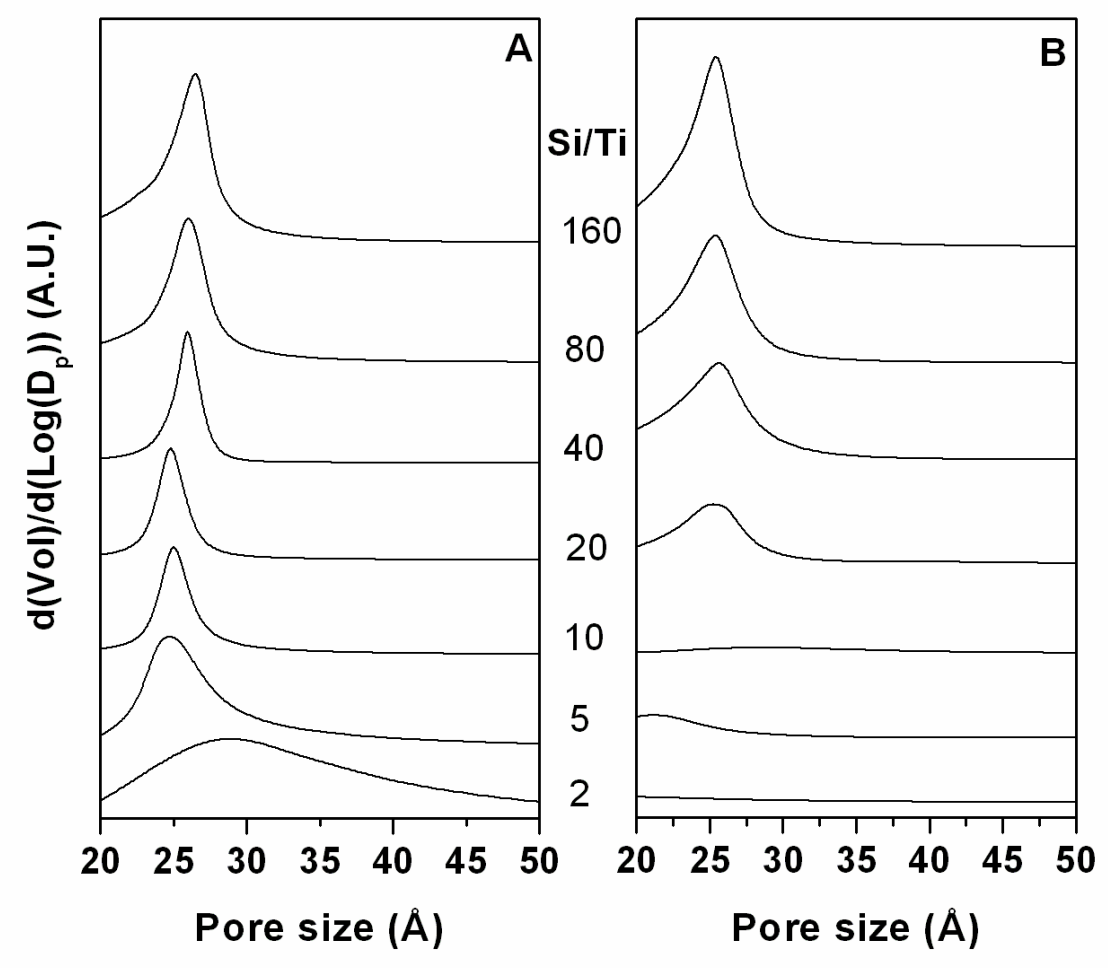

Figure 2

J. Iglesias et al. 

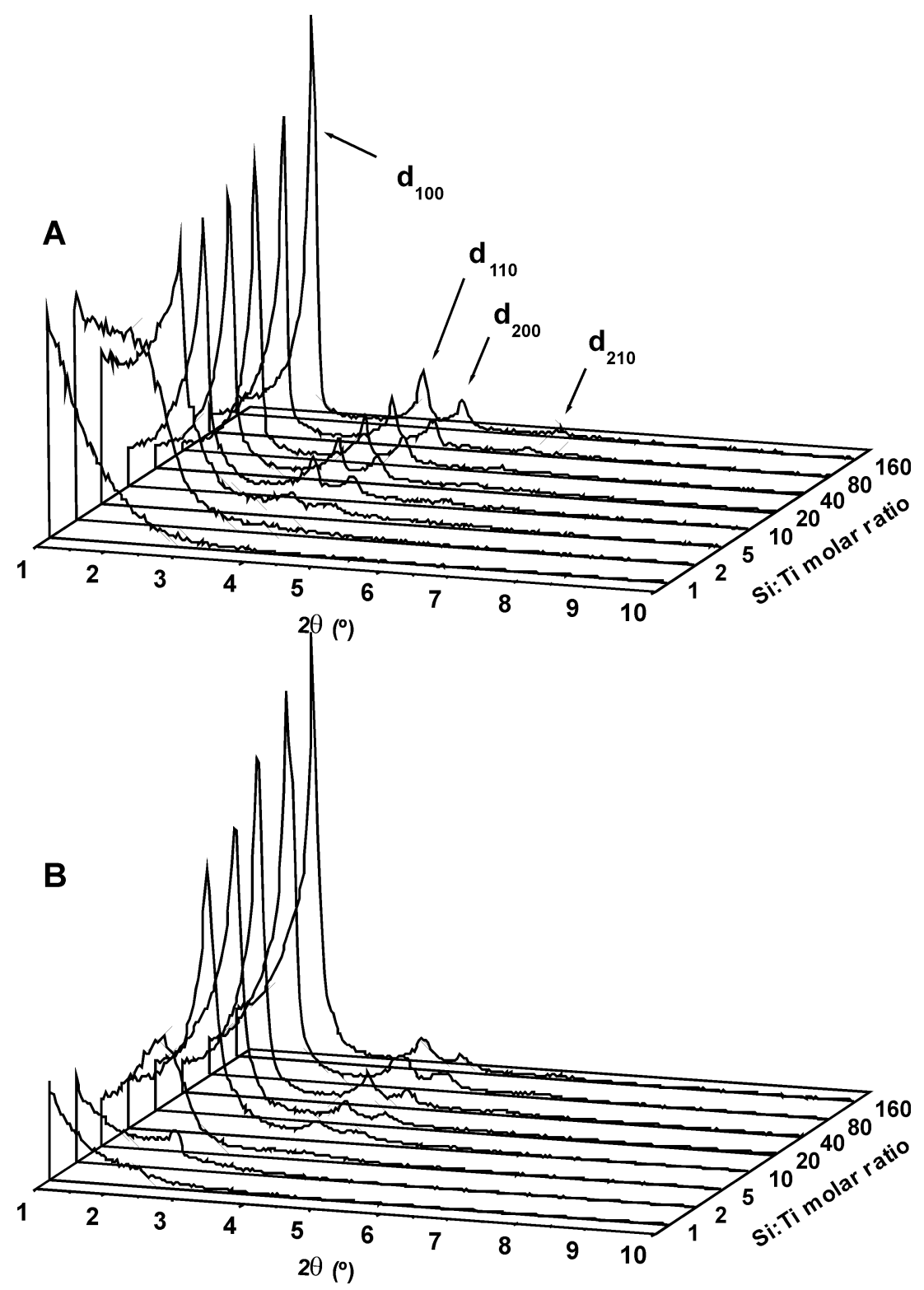

Figure 3

J. Iglesias et al. 


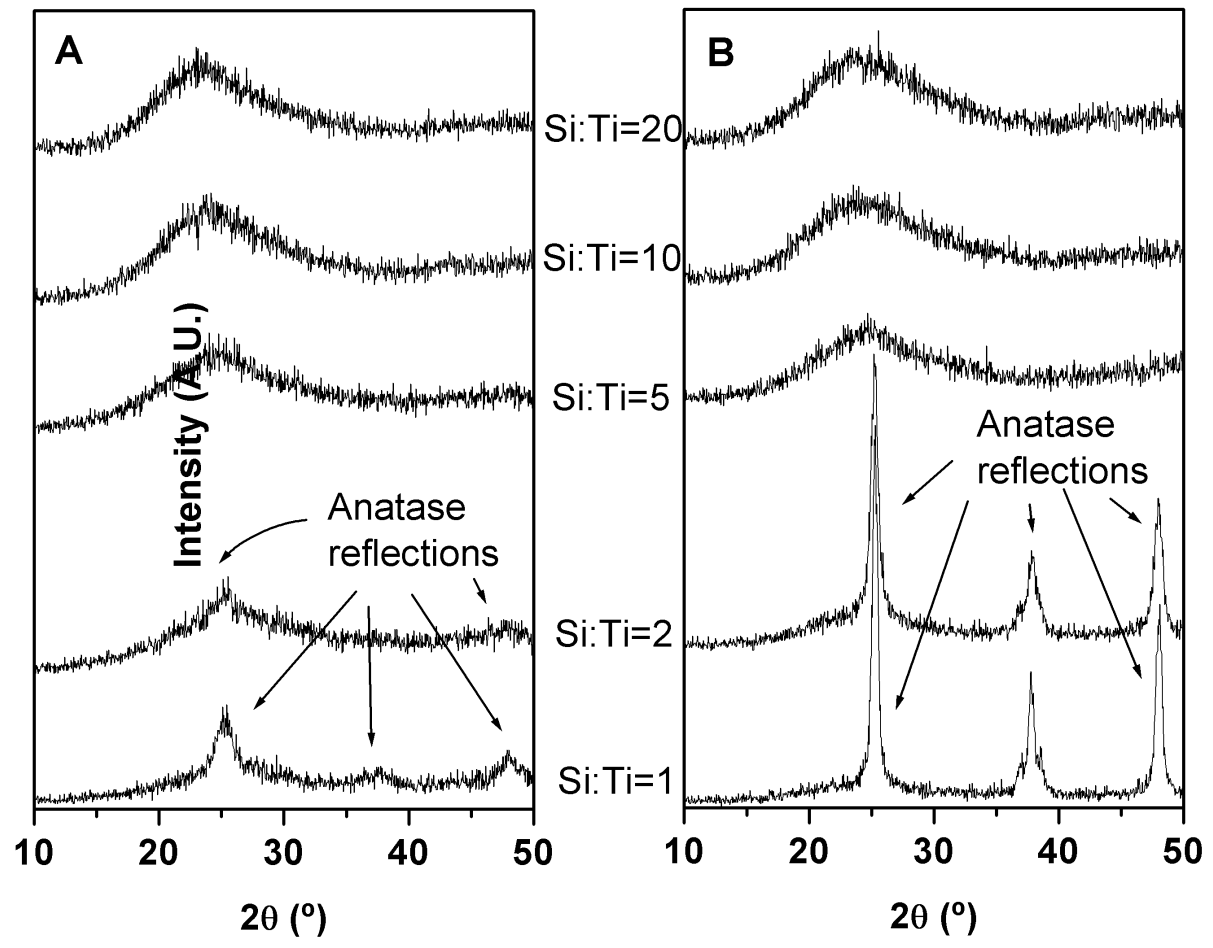

Figure 4

J. Iglesias et al. 

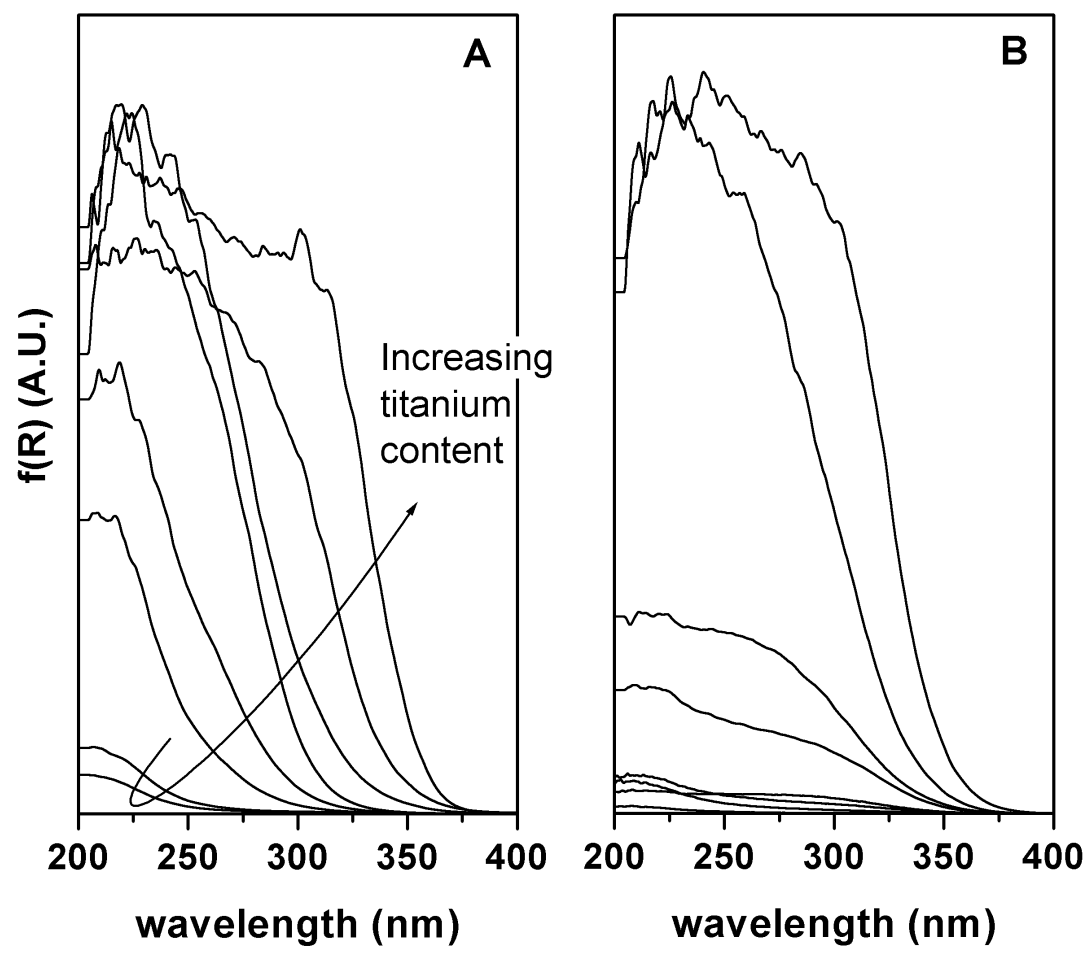

Figure 5

J. Iglesias et al. 


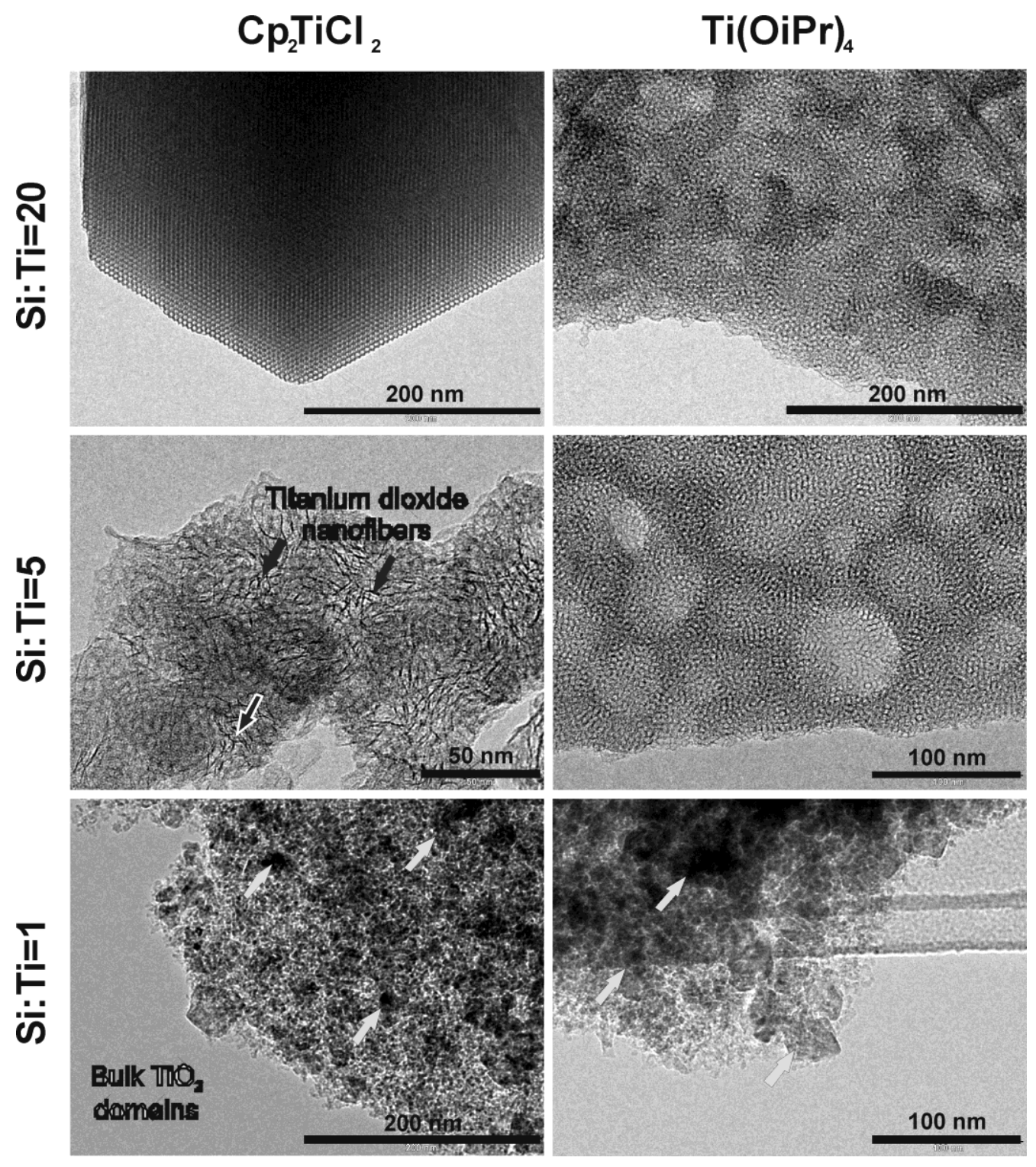

Figure 6

J. Iglesias et al. 


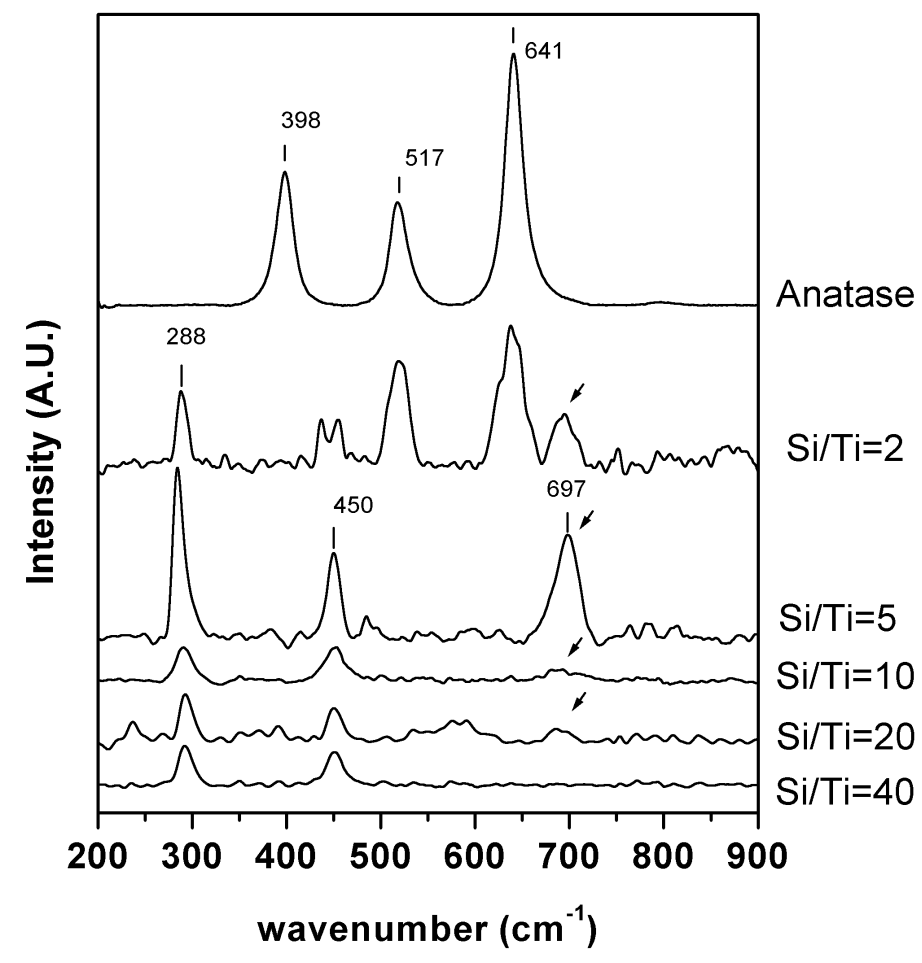

Figure 7

J. Iglesias et al. 
$\square$ Substrate Conversion (\%) $\square$ TBHP Efficiency (\%)
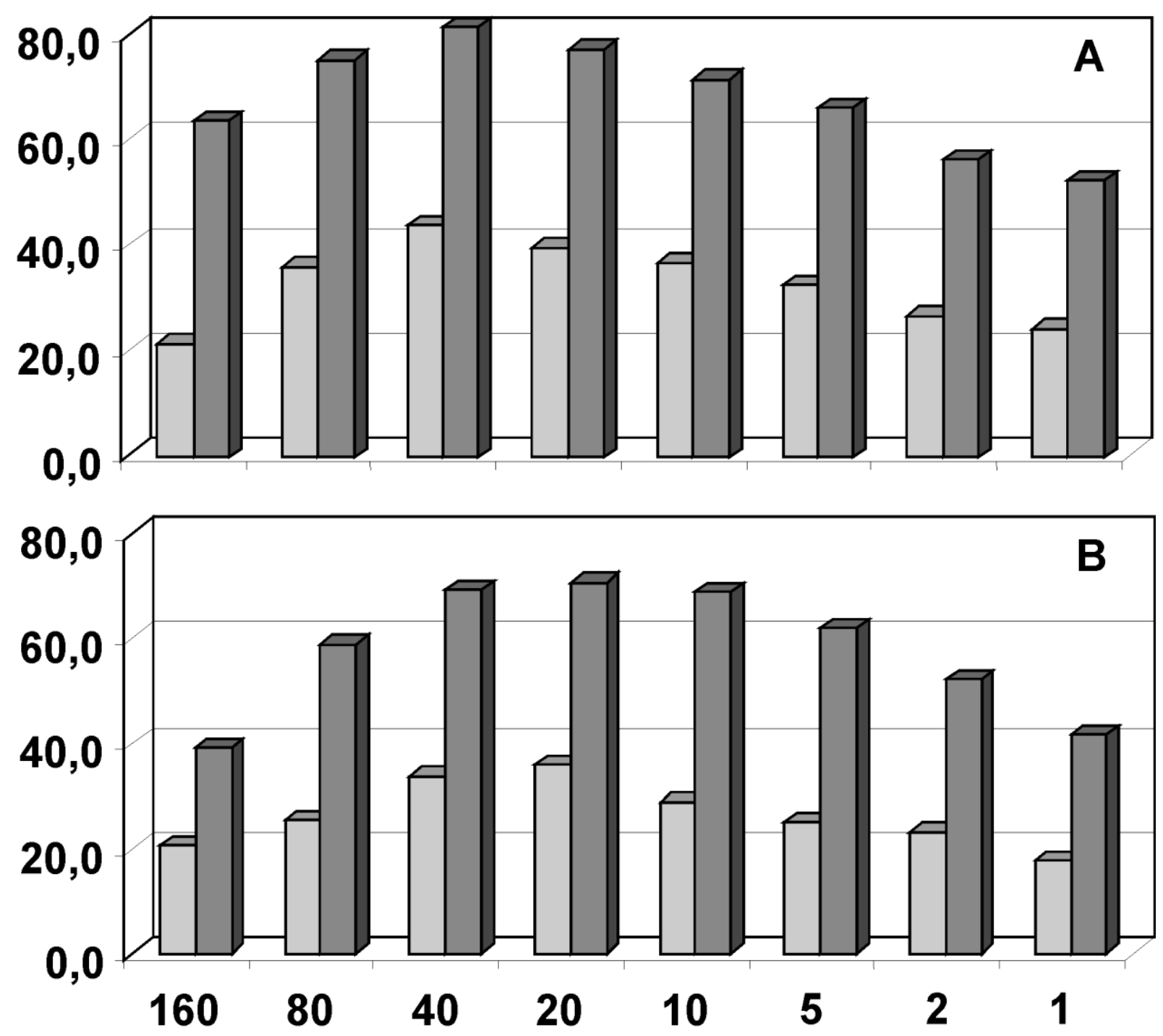

Si / Ti molar ratio

Figure 8

J. Iglesias et al. 

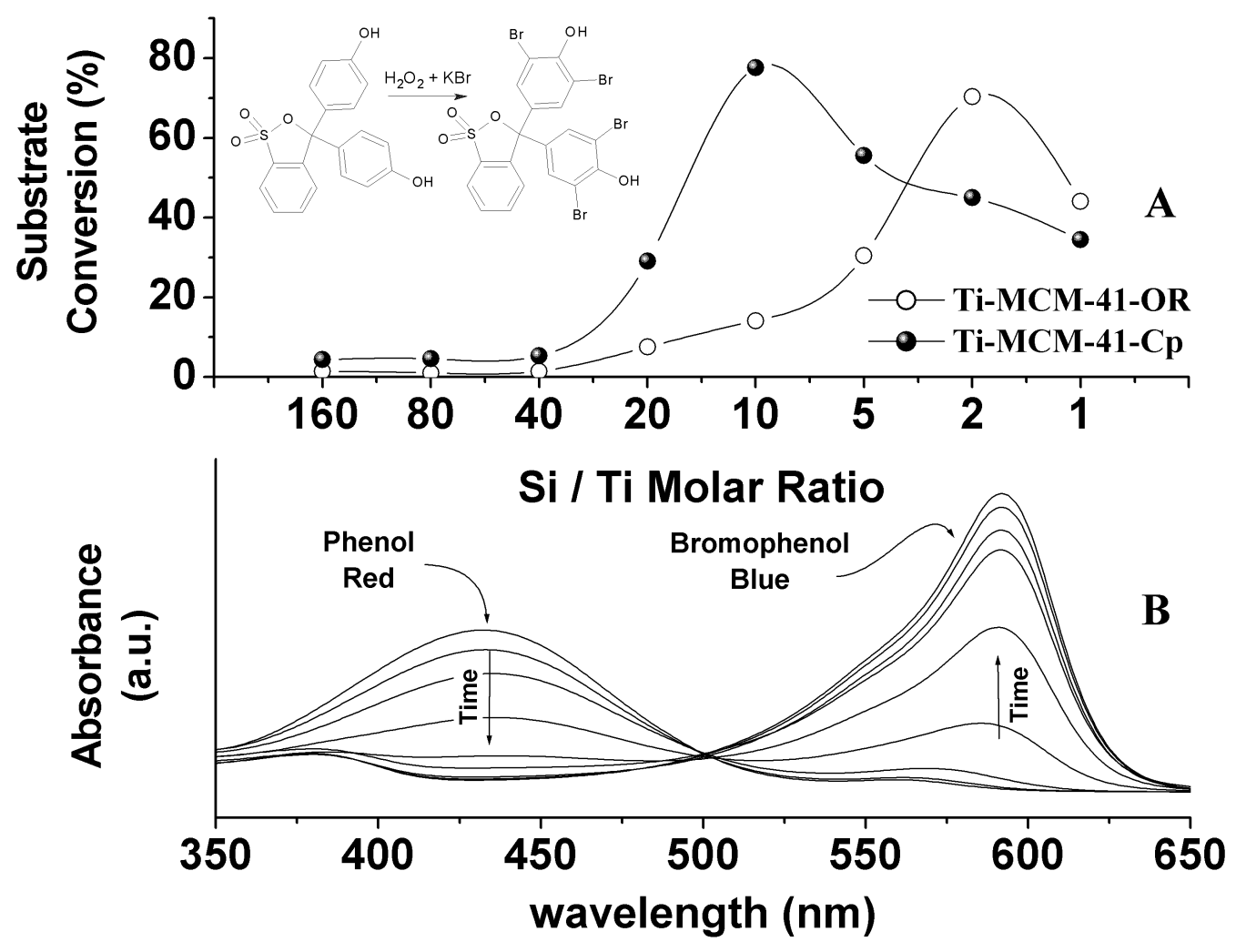

Figure 9

J. Iglesias et al. 

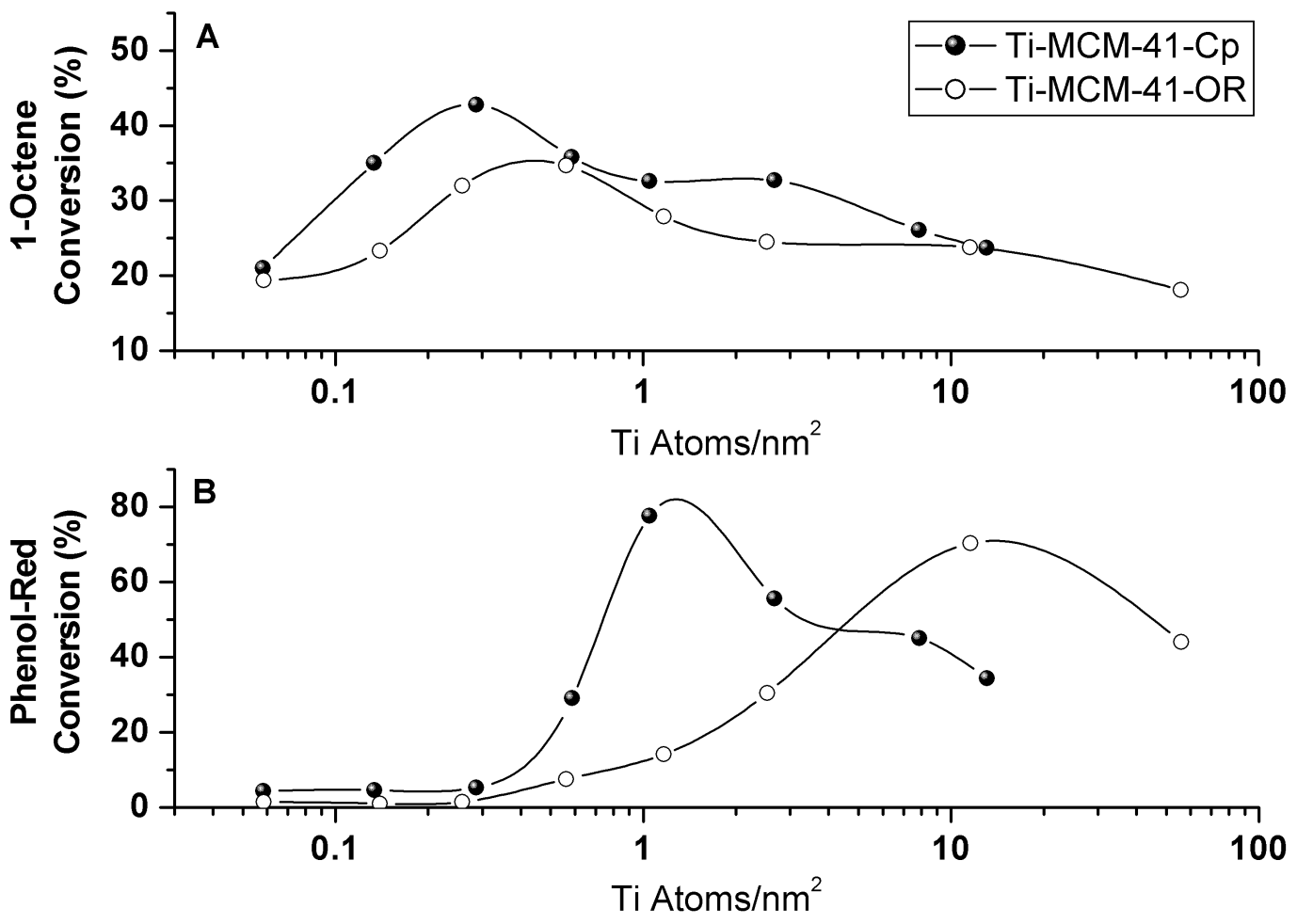

Figure 10

J. Iglesias et al. 
Table 1. Phyico-chemical properties of titanium-functionalized MCM-41 materials

\begin{tabular}{|c|c|c|c|c|c|c|c|c|}
\hline \multirow{2}{*}{$\begin{array}{c}\mathbf{T i} \\
\text { Source }\end{array}$} & \multicolumn{2}{|c|}{ Metal loading } & \multicolumn{3}{|c|}{ Textural properties } & \multicolumn{3}{|c|}{ Structural properties } \\
\hline & $\underset{(\text { molar) }}{\mathrm{Si} / \mathrm{Ti}^{\mathrm{a}}}$ & $\begin{array}{c}\mathbf{T i}^{\mathbf{b}} \\
(\% \mathbf{w t} .)\end{array}$ & $\begin{array}{c}\mathrm{Sg}^{\mathrm{c}} \\
\left(\mathbf{m}^{2} \cdot \mathrm{g}^{-1}\right)\end{array}$ & $\begin{array}{c}\mathbf{V p}{ }^{\mathbf{d}} \\
\left(\mathrm{cm}^{3} \cdot \mathrm{g}^{-1}\right)\end{array}$ & $\underset{(\stackrel{A}{)})}{\mathbf{D p}^{\mathrm{e}}}$ & $\begin{array}{l}d_{100}{ }^{f} \\
(\AA)\end{array}$ & $\begin{array}{l}\mathbf{a}_{0}{ }^{\mathrm{g}} \\
(\AA)\end{array}$ & $\begin{array}{l}\mathbf{W t}^{h} \\
(\AA)\end{array}$ \\
\hline \multirow{8}{*}{$\begin{array}{l}\underbrace{N} \\
\stackrel{\sigma}{\tilde{U}}\end{array}$} & 160 & 0.5 & 1081 & 0.89 & 27 & 42 & 48 & 21 \\
\hline & 80 & 1.1 & 1036 & 0.88 & 26 & 42 & 49 & 23 \\
\hline & 40 & 2.1 & 923 & 0.82 & 26 & 43 & 49 & 23 \\
\hline & 20 & 3.9 & 836 & 0.75 & 25 & 43 & 49 & 24 \\
\hline & 10 & 6.6 & 792 & 0.72 & 25 & 42 & 49 & 24 \\
\hline & 5 & 15.2 & 717 & 0.71 & 24 & 42 & 48 & 24 \\
\hline & 2 & 28.5 & 455 & 0.49 & 22 & --- & --- & --- \\
\hline & 1 & 36.3 & 350 & 0.57 & --- & --- & --- & --- \\
\hline \multirow{8}{*}{ 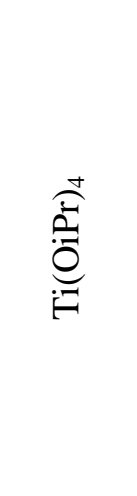 } & 160 & 0.5 & 1075 & 0.88 & 26 & 41 & 47 & 21 \\
\hline & 80 & 1.1 & 993 & 0.86 & 26 & 40 & 47 & 21 \\
\hline & 40 & 2.0 & 975 & 0.79 & 26 & 40 & 47 & 21 \\
\hline & 20 & 4.0 & 895 & 0.83 & 27 & 42 & 48 & 21 \\
\hline & 10 & 7.4 & 798 & 0.82 & 28 & 41 & 47 & 19 \\
\hline & 5 & 12.4 & 618 & 0.69 & 23 & 41 & 47 & 24 \\
\hline & 2 & 26.6 & 290 & 0.23 & 20 & --- & --- & --- \\
\hline & 1 & 35.5 & 80 & 0.08 & --- & --- & --- & --- \\
\hline
\end{tabular}

\footnotetext{
${ }^{a}$ Initial composition of the synthesis gel. ${ }^{b}$ Final titanium content after calcination. ${ }^{\mathrm{c}}$ Surface area estimated by the BET method. ${ }^{d}$ Total pore volume recorded at $\mathrm{p} / \mathrm{p} 0=0.985$. ${ }^{\mathrm{e}}$ Mean pore size evaluated by the $\mathrm{BJH}$ method applying the KJS correction. ${ }^{\mathrm{f}}$ Interplanar spacing calculated through the Bragg law. ${ }^{\mathrm{g}}$ Unit cell size calculated assuming hexagonal geometry. ${ }^{\mathrm{h}}$ Pore wall thickness calculated as $\mathrm{a}_{0}-\mathrm{Dp}$
} 